\title{
Developing Rehabilitation Robots for the Brain Injured
}

\author{
Paul Gnanayutham ${ }^{1}$ and Jennifer George ${ }^{2}$ \\ 'School of Computing, University of Portsmouth, Buckingham Building, \\ Lion Terrace, Portsmouth, PO1 3HE, United Kingdom, \\ 2 \\ Qantm College, SAE House, 297 Kingsland Road, E8 4DD London, United Kingdom, \\ ${ }^{1}$ paul.gnanayuthameport.ac.uk, ${ }^{2}$ jennifer.georgedsae.edu
}

\begin{abstract}
Although rehabilitation robotics have been used for helping disabled persons in various areas of disability, such as stroke rehabilitation, very little research has been done with the brain injured persons and robotics. This paper discusses the implementation of a simple model, which consists of brain body interface, a computer, an interface program and an electronic circuit to interface the computer to a robotic arm. This was an exploratory research that was carried out that allowed a brain-injured person to do simple tasks using robotic arms. This paper also looks at rehabilitation robotics both past and present. The paper goes on to explore the new avenues available to enhance this exploratory research. In this paper, we take the brain body interface communications a step further where the brain injured persons will not only communicate but will also be able to do simple tasks.
\end{abstract}

Keywords: Rehabilitation, Robot, Brain Injury, Cyberlink ${ }^{\mathrm{TM}}$, Brain Body Interface, Brain Computer Interface

\section{INTRODUCTION}

The word 'Robot' come originally from a play in 1923 with the meaning a 'slave worker' (from the 1923 play Rossum's Universal Robots). The early uses of robots were mechanical devices using gears and levers. The advent of computers and the fast and furious new technology has given the robots the capability to perform sophisticated tasks others than mundane routine jobs. Robots are in action in the Military, Health sector, Manufacturing, Space exploration, Mining etc. If asked to describe a robot many people would describe something from a science fiction, our mental images are guided more by science fiction than science reality. The reality is probably a car assembly robot rather than the evil Dalek of Dr Who. Originally most robots were used as tools in a manufacturing environment, whether spraying, welding or assembling. These are normally referred to as assembly robots. It has long been realised that, while robotics will continue to have a vital role in the manufacturing industry, the areas for growth lie in applications in "unstructured and hostile environments". Many applications in an unstructured environment are "service" applications. Service robots have for a while been used for tasks at home such as 
cleaning, cutting the lawn, etc. Also hazardous environments such as searching underwater, within a nuclear reactor, the making safe of bombs and mines.

Over the past 20 years there has been much research in the area of 'advanced robotics'. The precision of a medical robot is claimed to be even better than a skilled surgeon www.prosurgics.com. In addition to surgery, robotic technology can be used in several ways to benefit people with disabilities. Although there are many potential benefits for the disabled user, there are also very strong requirements and compromises, which must be considered in integrating the robotic arm with the wheelchair. Robots are being used in places such as Japan for caring for the physically handicapped people. These robots do the daily routine chaos thus taking the burden away from the careers and also saving a lot of money. The operators control these mobile robots through the Internet and mobile phones (Yoshiyuki et al., 2000). The new trend in robotics is to control robots remote using the Internet or mobile phone.

\subsection{Rehabilitation robotics from past to present}

Tzika and team claim (2008) that the brain has the ability to regain function through rehabilitative exercises following a stroke also that the brain is malleable, even six months or more after a stroke, which is a longer period of time than previously thought. This gives hope to people who have had strokes, their families and the rehabilitative specialists who treat those (Tzika et al., 2008).

The first referenced rehabilitation manipulator was the CASE manipulator built in the early 1960, which could move the user's paralysed arm (Kim and Cook, 1985). Mid 1970 saw the next stage of rehabilitation robotics in the form of a workstation based system designed by Roesler with five degree of freedom manipulator was placed in a specially adapted desktop environment, using rotating shelf units (Roesler et al., 1978). Another early workstation system was that of Seamone and Schmeisser. The arm of this system was based around an electrically powered prosthetic arm, mounted on a horizontal track. Various items of equipment (e.g. telephone, book rest, computer discs) were laid out on the simple but cleverly designed workstation table and could be manipulated by the arm using preprogrammed commands (Seamone and Schmeisser, 1986). Mason and Peizer (1978), developed the first ever robot arm mounted to a wheelchair, potentially offering much greater freedom than a workstation mounted system. The four degree of freedom arm and its novel telescoping design allowed it to reach to the floor or the ceiling (Hillman, 2003). Some more examples (Hillman et al., 2002a):

- Desk based system - "Wolfson" robot - for the desk based activities;

- Trolley mounted system - "Wessex" robot - requires a carer to move a trolley-based manipulator around from one room to another;

- Wheelchair mounted system - "Weston" robot - shares many common components with the trolley-mounted system, but mounted to a wheelchair.

All the rehabilitation robots mentioned above needed some type of motoring ability to manipulate such as a switch or joy stick and utilise the robot for carrying out various tasks. However the brain injured quadriplegic community was not catered by 
the developers of the rehabilitation robots above. This paper looks at the possibility of catering for this group of brain injured quadriplegic individuals to use rehabilitation robots for similar usage. The results of an exploratory study carried out using a robotic arm and bio-potentials from the forehead of a brain injured user is used an example of what could be done to cater for this group of brain injured quadriplegic individuals.

\section{DESIGNING FOR THE BRAIN INJURED}

There are several areas where a robotic device might assist a disabled person, here are some examples (Hillman et al., 2002b):

- Eating and drinking (Fig.1);

- Personal hygiene, such as washing, shaving;

- Work and leisure, such as handling papers, books or videos (Fig.2);

- Access, such as opening doors, operating light switches or lift buttons;

- Reaching and moving, such as reaching down to pick up an item off the floor or reaching up to get an item off of a shelf.

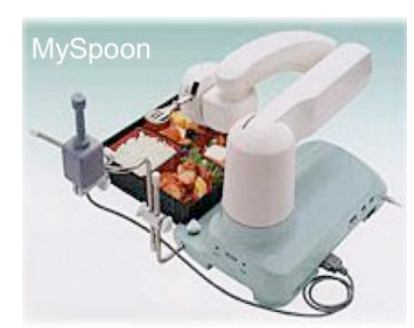

Fig. 1. Robot for eating and drinking

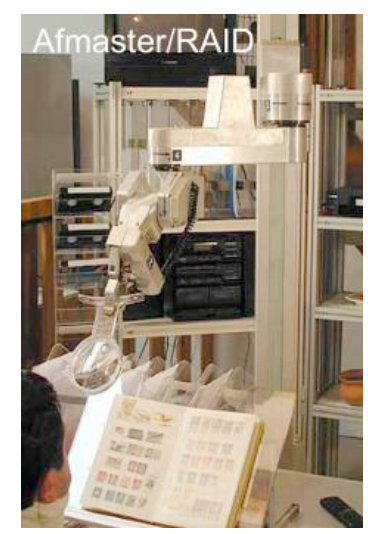

Fig. 2. Work and leisure, such as handling papers, books or videos 
As for the mechanical construction of attaching a robotic extension to a wheelchair there are different requirements in comparison to either a desktop or mobile system. Mounting a robotic extension into a desktop provides a "workstation" based approach, and all the items to be manipulated are placed within a known area of reach. The mobile approach is similar since the trolley-mounted robotic extension is intended to interact with objects at a number of different workstations (Hillman et al., 2002b). Another choice of construction will be an agent architecture in which the agent must operate without hindering the user's ability to take direct action when he/she will choose (Hillman et al., 2002b).

\section{ROBOTS FOR QUADRIPLEGIC BRAIN INJURED}

Not all users with special needs can use a mouse, trackball, and keyboard or have the ability to speak to a speech recognition system. So we need a device that provides communication capabilities for those who cannot use any of the regular input devices (Gnanayutham and George, 2007 and 2009) such as:

- HeadMouse ${ }^{\mathrm{TM}}$ - using wireless optical sensor that transforms head movement into cursor movement on the screen;

- Tonguepoint ${ }^{\mathrm{TM}}$ - a system mounted on mouth piece;

- Eye TrackerTM - a system that allows the monitoring of both the conscious and unconscious gaze

All the devices above have their advantages and disadvantages. A user with cerebral palsy will not have good motor abilities to operate the 'Tonguepoint ${ }^{\mathrm{TM}}$ '. A user with spinal vertebrate fusion may not be able to turn his or head and the HeadMouse ${ }^{\mathrm{TM}}$ will be of no use to this user.

Fig. 3. Cyberlink

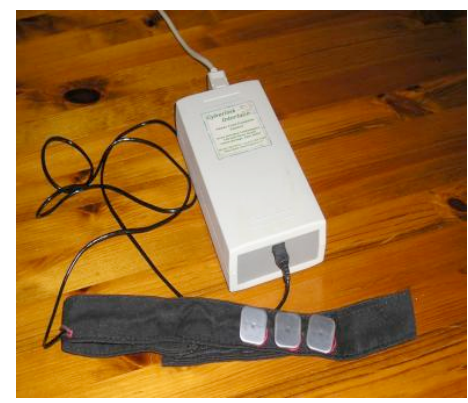

At present one of the brain computer interface devices that can cater for the quadriplegic brain injured individual is the Cyberlink ${ }^{\mathrm{TM}}$ (Fig. 3) because it uses a combination of bio-potentials. 'Cyberlink ${ }^{\mathrm{TM}}$ ' can be used as a technology that combines eye movement, eye blink, facial muscle and brain wave bio-potentials detected at the user's forehead to generate a mouse input that can be used for communicating. Cyberlink ${ }^{\mathrm{TM}}$ uses the forehead as noninvasive site, for convenience and also because it has a rich variety of bio-potentials. The signals for communications are obtained by attaching probes on the forehead of the patients. It is 3 silver/silver chloride contact electrodes (i.e. non-invasive), which are placed on a 
headband that picks up EEG (brain wave), EMG (muscle movement wave) and EOG (Eye ball movement) signals when applied on the forehead. These are then fed into an amplifier box and then to the mouse port, so the computer just sees the device as a mouse, which, is used to control the cursor. The main signals used are due to muscle movement, only about $10 \%$ is due to thought processes (Brain wave). We used the Cyberlink $^{\mathrm{TM}}$ to communicate with the brain injured persons to get basic yes/no answers (Gnanayutham and George, 2009a). This time we want to go a step further and make the brain injured user perform simple tasks using a robotic arm (Doherty et al., 2003, Gnanayutham et al., 2001).

The model for operating the robotic arm using the brain body interface consisted of following components:

1. A Cyberlink ${ }^{\mathrm{TM}}$ brain body actuated control technology system that connects to the computer via the serial port;

2. A computer with a parallel port and serial port free. An Interface program written in Visual Basic ${ }^{\mathrm{TM}}$ to operate the functions of the robotic arm;

3. An Electronic circuit to read the parallel port of the computer and operate the motors that manipulate the robotic arm;

4. A robotic arm (Super Armatron ${ }^{\mathrm{TM}}$ ) that is operated using a series of motors.

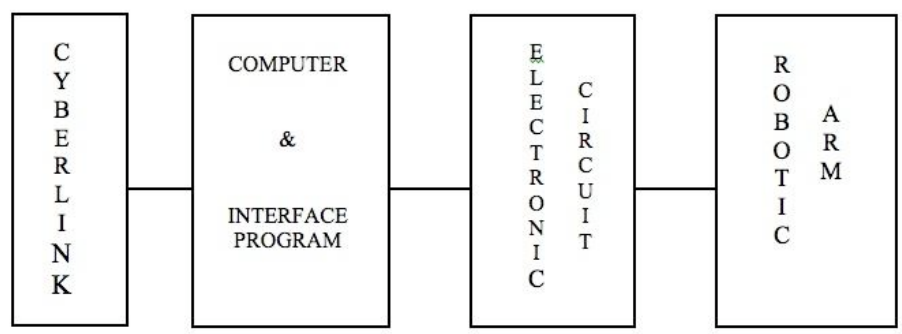

Fig. 4. Model for operating the robotic arm

The fig. 4, shows the model for operating the robotic arm using the brain body interface. The computer needed one serial port for the Cyberlink ${ }^{\mathrm{TM}}$ and a parallel port for the electronic circuit that interfaced the computer with the robotic arm. The Cyberlink probes were attached to the forehead of the user and the other end of the Cyberlink was connected to the serial port of the computer (Gnanayutham et al., 2001). 


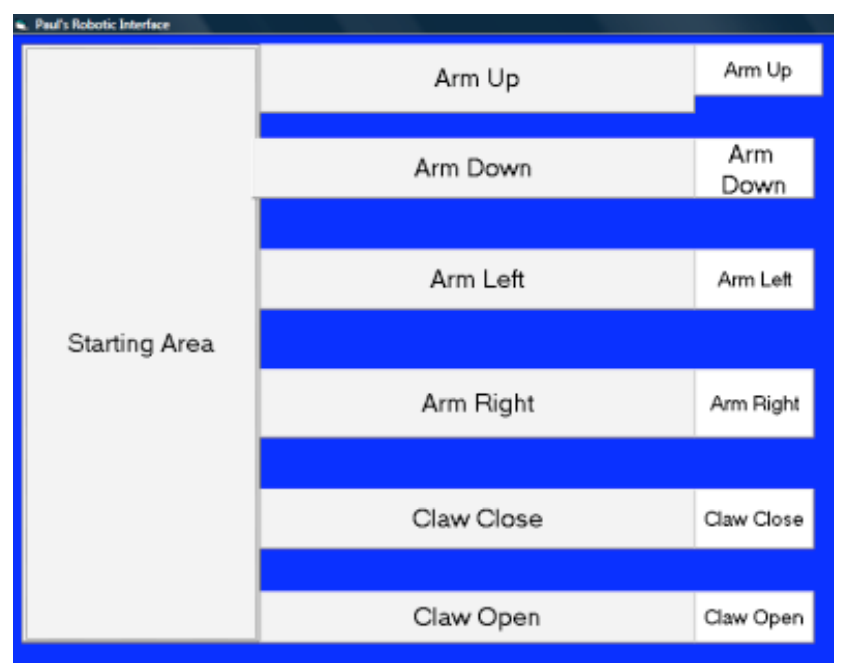

Fig. 5. Interface Program

The computer had a program written in Microsoft Visual Basic $6.0^{\mathrm{TM}}$, which had six paths for controlling the robotic arm (Fig. 5). The paths ended up in one of these functions, arm go up, arm down, arm left, arm right, open claw and close claw. When one of these six functions were triggered, the program sent a binary code to the parallel port, which drove one of the motors to carry out what was requested by the user. The Electronic circuit used in the above setup is shown below.

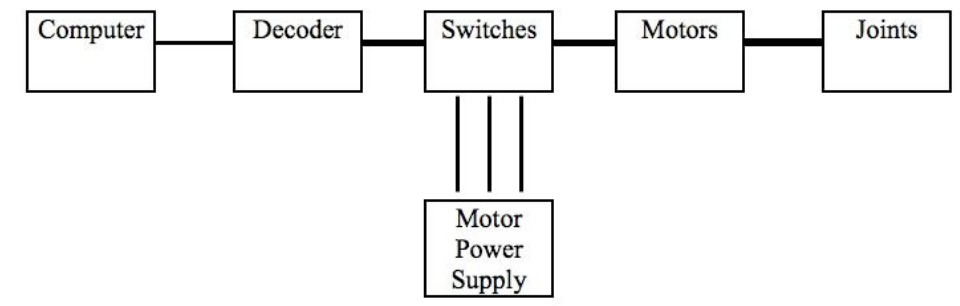

Fig. 6. Electronic Circuit

In Fig. 6, we see the block diagram of the electronic circuit that was used. The output from the parallel port was decoded and used for switching transistors. The transistors switched the motors on and off in either direction. The mechanical side of the circuit included aligning shafts and making sure there were no vibrations.

The robotic arm described in this section was developed and used as a live demonstration at the ICCIT'2001 conference in New Jersey where a quadriplegic individual picked up a cup to his mouth to show, how we can use a brain body interface and operate a robotic arm. Since then the researchers have concentrated on mainly communication, recreation and controlling the environment using brain body interfaces (Gnanayutham et al., 2009b). Now the researchers feel it's time to help this group of the disabled who perhaps need robotics than other motor impaired 
individuals. There is one previous successful research carried out by Felzer, and Freisleben (2002) but no commercial product has come out of that piece of research. At present work is carried out at University of Essex also on navigating a wheel chair using bio-potentials (Chen et al., 20007, Lai et al., 2009).

\section{SUMMARY AND CONCLUSIONS}

This paper indicated the various choices of rehabilitation robots that are available in the market, used mainly for stroke rehabilitation and other disability where the user is able manipulate a mouse, switch or a joystick. This paper also tackled the area of giving this sophisticated and powerful robot as a tool for the brain injured who perhaps need it more than any other category of people. Robots have been in science fiction for many years and but now we can extend these robots to the brain injured quadriplegics personnel by using their bio-potentials as the input instead of a mechanical switch, mouse or a joy stick. This exciting new research is going to change many a brain-injured person's life and set them on the path of rehabilitation.

\section{References}

1. Chun, S. L. T., Pei, J., John, Q. G., Huosheng, H., Kui, Y., (2007), EMG-based Hands-Free Wheelchair Control with EOG Attention Shift Detection, Proceedings of the 2007 IEEE International Conference on Robotics and Biomimetics, Sanya, China

2. Doherty, E., Wong, Y., Fernandes, J., Gnanayutham, P., Stephenson G., (2003). The OWI-007 Robotic Arm, Cyberlink Interface, and Visual $\mathrm{C}_{++}$Interface are a Great Help to the Handicapped and a Good Platform for Teaching Technology, Njedge.net Conference, October 2003, New York.

3. Felzer, T., Freisleben, B., (2002), HaWCoS: the "hands-free" wheelchair control system, Proceedings of the fifth international ACM conference on Assistive technologies, Edinburgh.

4. Gnanayutham, P., Bloor, C., Cockton, G. (2001). Robotics for the brain injured: An interface for the brain injured person to operate a robotic arm - October 2001, ICC/T'2001, New York.

5. Gnanayutham, P., George, J., (2007). Inc/usive Design for Brain Body Interfaces, Edited by Schmorrow, D., D and Reeves, L., M., HCI International 2007, Lecture Notes in Computer Science Series, Springer, LNAI 4565, July 2007, Beijing, $102-111$.

6. Gnanayutham, P., George, J., (2009a). Communicating, Recreating and Controlling the Environment Using an Adaptive Brain-Body Interface, DSAI 2009, June 2009, Published by UTAD, Lisbon, 71 - 78.

7. Gnanayutham, P., Cockton, G., (2009b). Adaptive Personalisation for Researcher-Independent Brain-Body Interface Usage, CHI 2009, April 2009, ACM Press, Boston, 3003 - 3019. 
8. Hillman, M.R., (2003). Rehabilitation robotics from past to present - a historical perspective, ICORR, April 2003, Daejeon.

9. Hillman, M.R., Evans, N.M., Orpwood, R.D., (2002a). Improving the flexibility of an assistive Robot, Proceedings of the Cambridge Workshop on Universal Access and Assistive Technology 2002, Springer Verlag.

10. Hillman, M.R., Hagan, K., Hagan, S., Jepson, J., Orpwood, R.D., (2002b). The Weston Whee/chair Mounted Assistive Robot - The Design Story, Robotica Vol. 20, 125 - 132, Cambridge University Press

11. Lai, W., Huosheng, H., Kui, Y., (2009), Use of Forehead Bio-signals for Controlling an Intelligent Wheelchair, Proceedings of the 2008 IEEE, International Conference on Robotics and Biomimetics, Bangkok.

12. Mason, C.P., E.Peizer, E., (1978). Medical Manipulator for Quadriplegic, IRIA 1978, New Delhi.

13. Roesler.H., Kuppers, H.J., Schmalenbach. E. (1978). The Medical Manipulator and its Adapted Environment: A System for the Rehabilitation of Severely Handicapped, Proc Int'l Conf. on Telemanipulators for the Physically Handicapped.

14. Seamone, W., Schmeisser, G., (1986). Evaluation of the JHU/APL Robot Arm Workstation, Interactive Robotic Aids. World Rehabilitation Fund Monograph\#37, York.

15. Tzika, A., Khanicheh, A., Rosen, B., Astrakas, L., Moskowitz, M., (2008). Stroke Rehabilitation Improved By Robotic Technology, Medical News Today, December 2008.

16. Y.Kim, Y., Cook, A.M., (1985). Manipulation and Mobility Aids, Electronic Devices for Rehabilitation, Chapman and Hall, London.

17. Yoshiyuki, T., Takashi, K., Tateki, U., Masao, M., Hiroyuki, K., (2000). Development of the Mobile Robot System to aid the daily life for Physically Handicapped, Proceedings of the $7^{\text {th }}$ International Conference on Computers Helping People with Special Needs, Boston. 